Enhanced i nt er nal i zat i on of macromol ecul ar drugs i nt o Mycobact er i um snegmat is wi th the assi st ance of si I ver nanoparti cl es

\begin{tabular}{|c|c|}
\hline 著者 & $\begin{array}{l}\text { Sun Fangf ang, Oh Sangj i n, K m Jeonghyo, Kat o } \\
\text { Tat suya, K m Hwa J ung, Lee J aebeom Park Enoch } \\
\text { Y. }\end{array}$ \\
\hline $\begin{array}{l}\text { j our nal or } \\
\text { publ i cat i on } \mathrm{titl} \text { e }\end{array}$ & Journal of mi crobi ol ogy and bi ot echnol ogy \\
\hline vol une & 27 \\
\hline nunber & 8 \\
\hline page $r$ ange & $1483-1490$ \\
\hline year & $2017-08-28$ \\
\hline 出版者 & $\begin{array}{l}\text { The Kor ean Soci et y for M crobi ol ogy and } \\
\text { Bi ot echnol ogy. }\end{array}$ \\
\hline 権利 & $\begin{array}{l}\text { Copyri ght ( C) } 2017 \text { by the Kor ean Soci ety for } \\
\text { M crobi ol ogy and Bi ot echnol ogy. Al I ri ght } \\
\text { reserved. }\end{array}$ \\
\hline URL & ht t p: //hdl . handl e. net /10297/10378 \\
\hline
\end{tabular}




\section{Enhanced internalization of macromolecular drugs into Mycobacterium smegmatis with the assistance of silver nanoparticles}

Fangfang Sun ${ }^{1,2, \dagger}$, Sangjin $\mathrm{Oh}^{3, \dagger}$, Jeonghyo Kim ${ }^{3}$, Tatsuya Kato ${ }^{1}$, Hwa-Jung $\mathrm{Kim}^{4}$, Jaebeom Lee $^{3 *}$, Enoch Y. Park ${ }^{1 *}$

${ }^{1}$ Research Institute of Green Science and Technology, Shizuoka University, 836 Ohya, Suruga-ku, Shizuoka 422-8529, Japan

${ }^{2}$ Department of Biomedical Engineering, College of Life Information Science and Instrument Engineering, Hangzhou Dianzi University, Hangzhou 310018, China

${ }^{3}$ Department of Cogno-mechatronics Engineering, Pusan National University, Busan 46241, Republic of Korea

${ }^{4}$ Department of Microbiology and Research Institute for Medical Science, College of Medicine, Chungnam National University, Daejeon 35015, Republic of Korea

sunff511@hdu.edu (FS) ; ohsj1658@ naver.com (SO) ; yaamako@ gmail.com (JK) ;

kato.tatsuya@ shizuoka.ac.jp (TK); hjukim@cnu.ac.kr (HJK)

*Corresponding authors: jaebeom@pusan.ac.kr (JL) ; park.enoch@ shizuoka.ac.jp (EYP)

$\dagger$ Both contributes equally. 


\begin{abstract}
In this study, silver nanoparticles (AgNPs) were synthesized by the citrate reduction process and, with the assistance of $n$-hydroxysuccinimide and 1-ethyl-3-(3-dimethylaminopropyl) carbodiimide, were successfully loaded with the macromolecular drug vancomycin (VAM) to form AgNP-VAM bioconjugates. The synthesized AgNPs, VAM, and AgNP-VAM conjugate were characterized by UV-visible spectroscopy, zeta potential analysis, confocal microscopy, and transmission electron microscopy. The effect of loading VAM onto AgNPs was investigated by testing the internalization of the bioconjugate into Mycobacterium smegmatis. After treatment with the AgNP-VAM conjugate, the bacterial cells showed a significant decrease in UV absorption, indicating that loading of the VAM on AgNPs had vastly improved the drug's internalization compared with that of AgNPs. All the experimental assessments showed that, compared with free AgNPs and VAM, enhanced internalization had been successfully achieved with the AgNP-VAM conjugate, thus leading to significantly better delivery of the macromolecular drug into the $M$. smegmatis cell. The current research provides a new potential drug delivery system for the treatment of Mycobacterial infections.
\end{abstract}

Key Words: Mycobacterium smegmatis, silver nanoparticle, internalization, vancomycin, bioconjugate 


\section{Introduction}

Nanotechnology has provided a huge advantage to pharmacology through the design of drug delivery systems that are able to target phagocytic cells infected by intracellular pathogens, such as mycobacteria [1] . In addition, nanomaterials have been widely explored for the treatment [2], diagnosis [3], and monitoring of biological systems [4], including the development of nanoparticles for diagnostic and screening purposes, manufacture of drug delivery systems [5,6], and single-virus detection [7]. Of all the different kinds of metallic nanoparticles developed, silver nanoparticles (AgNPs) have attracted significant attention and interest for their potential applications in biomedicine as well as antibacterial devices [8-10]. In particular, AgNPs are known to be an effective bactericide against as many as 16 bacterial species [11-13]. Moreover, AgNPs can be readily chemically conjugated with a large number of biomolecules, such as proteins [14] and oligonucleotides [15,16], for improving their efficiency, with the advantage being that the biological properties of the conjugated materials remain unchanged [17]. Thus, bioconjugation with AgNPs has found new and wide applications in the fields of biosensing [18], DNA detection [16], and drug delivery [9]. For example, Thompson et al. [16] reported the first use of oligonucleotide-AgNP conjugates in a sandwich assay for targeted DNA detection, which enabled highly improved sensitivity compared with the conventional AuNP-based assay. Remarkably, the most highlighted application of AgNPs in the biological field is of antibacterial activity [19]. For example, Aymonier et al. [20] demonstrated that the hybrid of AgNPs and highly branched macromolecules exhibited environmentally friendly antimicrobial properties.

Numerous synthetic approaches have been developed for preparing the AgNPs, using templates [21], photochemistry [22, 23], electrochemistry [24, 25], and radiolysis [26, 27]. The simplest and most commonly used method for the synthesis of metal NPs is chemical reduction 
[28]. Generally, the synthesized AgNPs should have a controllable size and possess enough stability in aqueous state. In particular, AgNPs need to be able to present functional groups on their surface in order to conjugate with biomolecules. In this study, we have adopted a simple and rapid one-step synthesis method for the preparation of AgNPs by using the direct citrate reduction of silver nitrate under heat, via the well-known Turkevich method [29]. In this case, citrate serves dual roles as the reducing and capping agent. As citrate is characterized by its many hydroxyl groups, its capping role as the nanoparticle stabilizer would impart many hydroxyl groups on the surface of the AgNPs. This aid the molecular loading, allowing molecules possessing amine and other functional groups to form hydrogen bonds with the AgNPs, thereby facilitating the bioconjugation process. Furthermore, it has been reported that the charged citrate coating improved stability of Ag NPs and decreased their toxicity [30]. If the NP size is greater than $15-20 \mathrm{~nm}$, it is not considered an important parameter to determine its biological effect [31] [32].

Vancomycin (VAM) is a widely used glycopeptide antibiotic. Ever since the spread of antibiotic resistance in the 1980s [33], VAM has become an essential antibiotic and has come into widespread use owing to its capability of inhibiting the growth of a broad range of Grampositive bacteria $[2,34]$. Nevertheless, VAM is a hydrophilic molecule of bulk size, which may restrict its transport through the bacterial cell membrane, thus leading to its low internalization ability. Based on this fact, we developed AgNP-VAM conjugates for studying the enhanced internalization of macromolecular drugs by AgNPs.

Therefore, in this study, we used citrate as the reductant and stabilizer simultaneously for the synthesis of AgNPs. With the assistance of $n$-hydroxysuccinimide (NHS) and 1-ethyl-3-(3dimethylaminopropyl) carbodiimide (EDC) (Fig. 1A), VAM was successfully loaded onto the surface of the AgNPs (Fig. 1B). The free AgNPs, VAM, and AgNP-VAM conjugate were 
characterized in terms of their physicochemical and photonic properties. Moreover, the antimicrobial effect of the AgNP-VAM conjugate was tested on Mycobacterium smegmatis. With the assistance of the AgNPs, enhanced internalization of VAM was successfully achieved, thus leading to significantly better delivery of the antibiotic into the bacterium. Based on the results, the current research may provide a new drug delivery system for the treatment of Mycobacterial infections.

\section{Experiments}

\subsection{Materials}

Silver nitrate $\left(\mathrm{AgNO}_{3}, 99.99 \%\right), 8$-mercaptootanoic acid (8-MOA), NHS, EDC, and VAM were purchased from Sigma-Aldrich (Milwaukee, WI). Trisodium citrate dihydrate was obtained from Wako Pure Chem. Ind. Ltd. (Osaka, Japan). All the reagents were used as received without further purification. Mycobacterium smegmatis, which was purchased from the Biological Resource Center (Kisarazu-shi, Chiba, Japan), was cultivated at $37^{\circ} \mathrm{C}$ and maintained on LuriaBertani (LB) nutrient agar plates.

\subsection{Preparation of AgNPs and AgNP-VAM conjugates}

AgNPs of approximately $17 \mathrm{~nm}$ in size were prepared by citrate reduction of silver nitrate. In brief, $30 \mathrm{~mL}$ of $1 \mathrm{mM} \mathrm{AgNO}$ was brought to reflux while stirring, and $5 \mathrm{~mL}$ of a $38.8 \mathrm{mM}$ trisodium citrate solution was then added quickly, which resulted in a color change of the solution from colorless to bright yellow within $5 \mathrm{~min}$. The reaction mixture was heated for an additional $4 \mathrm{~min}$, allowed to cool to room temperature, and subsequently filtered through a Millex-GP 0.22- $\mu$ m filter (Millipore Corp., Billerica, MA, USA). A typical solution of 17-nmdiameter AgNPs exhibited a characteristic surface plasmon band centered between 416 and 
$420 \mathrm{~nm}$. The AgNPs were further modified by 8-MOA, following which $200 \mu \mathrm{L}$ of $0.01 \mathrm{M}$ NHS was added to $10 \mathrm{~mL}$ of the prepared 0.01 M AgNP-MOA. After stirring for $1 \mathrm{~min}, 200$ $\mu \mathrm{L}$ of $0.01 \mathrm{M}$ EDC was added and the mixture was stirred for another 10 min. Finally, the AgNP-VAM conjugate was obtained by adding $1 \mathrm{~mL}$ of $2.5 \mathrm{mM}$ VAM into the mixture and stirring it for 30 min. Figure 1A shows a schematic representation of the AgNP modification process. To verify internalization capacity of AgNPs and AgNP-VAM conjugates into the cells, M. smegmatis cells were cultured for $24 \mathrm{~h}$ with prepared materials. To measure the remained AgNPs and AgNP-VAM conjugates in the culture medium, some part of solution was taken and centrifugated from cells. Finally separated solution was measured by real-time absorption intensities.

\subsection{Viability test}

M. smegmatis cells were cultured overnight in Luria-Bertani (LB) medium on a rotary shaker at $140 \mathrm{rpm}$ at $37^{\circ} \mathrm{C}$. Bacterial cells at the stationary growth phase were then diluted to a cell density of approximately $10^{6} \mathrm{CFU} / \mathrm{mL}$ with LB medium. To examine the growth kinetics of $M$. smegmatis in the presence of the various biomaterials, different doses of VAM, AgNPs, and AgNP-VAM in $15 \mathrm{~mL}$ of $\mathrm{LB}$ medium were incubated with the cells at $37^{\circ} \mathrm{C}$, with an agitation rate of $140 \mathrm{rpm}$.

\subsection{Analytical methods}

Absorption spectra of the AgNPs and AgNP-VAM were obtained by UV-visible (UV-vis) spectroscopy (S31500; SCINCO, Seoul, Korea). The size of the nanoparticles was measured with a Zetasizer (Nano-ZS; Malvern, Malvern, UK). Fourier transform infrared spectrophotometry (FTIR 8700; Shimadzu, Kyoto, Japan) was used to confirm the chemical bonding between the VAM and AgNPs. Morphological analysis of the nanoparticles was 
carried out by confocal laser scanning microscopy (LSM 700; Carl Zeiss Microimaging GmbH, Jena, Germany) and scanning transmission electron microscopy (STEM; JEM, Akishima, Japan). Samples for TEM studies were prepared by placing one drop of the AgNP and AgNPVAM solutions on carbon-coated TEM grids (Okenshoji, Tokyo, Japan), drying the grids at room temperature, and observing them at $150 \mathrm{~K} \times$ magnification in the microscope operated at $120 \mathrm{kV}$. The optical density of the M. smegmatis cells was investigated by UV-vis spectrophotometry (UV Mini 1240; Shimadzu, Kyoto, Japan) at $600 \mathrm{~nm}$. Samples were diluted if the optical density exceeded the measurement capability of the spectrophotometer. Quantification of the biomaterial-treated M. smegmatis was carried out by counting the viable colonies on LB agar plates and was expressed as colony-forming units per milliliter (CFU/mL).

\section{Results and Discussion}

\subsection{Characterization of the AgNPs and AgNP-VAM conjugates}

AgNPs were synthesized by the reduction of citrate (which served a dual role as the reducing and stabilizing agent), with the assistance of EDC and NHS, allowing a large amount of the drug to be loaded onto the surface of the AgNPs via strong amide bond formation [35]. As shown in Figure 2, the synthesized AgNPs exhibited a specific surface plasmon band at 420 nm, and AgNP-VAM conjugates shows plasmon peak at $430 \mathrm{~nm}$. TEM images (Fig. 2C and 2D) revealed the AgNPs to be well dispersed, with a size of $17 \pm 3 \mathrm{~nm}$, whereas the AgNPVAM conjugates had a larger size of approximately $30 \pm 3 \mathrm{~nm}$.

\subsection{FTIR analysis of the AgNPs and AgNP-VAM conjugates}


To confirm the structure of VAM after its bioconjugation with the AgNPs, FTIR spectra of the free AgNPs and VAM, and AgNP-VAM conjugate were recorded over a range of 4000-800 $\mathrm{cm}^{-1}$. The FTIR spectrum of VAM showed peaks at 3301 and $1661 \mathrm{~cm}^{-1}$ (Fig. 3A), which were assigned to the phenolic $\mathrm{OH}$ group and the aromatic $\mathrm{C}=\mathrm{C}$ stretching bond, respectively. The peak at $1498 \mathrm{~cm}^{-1}$ corresponded to the $\mathrm{C}=\mathrm{O}$ stretching bond, and the peak between 900 and $1350 \mathrm{~cm}^{-1}$ corresponded to the C-O stretching vibration mode [36]. In the case of the AgNPs synthesized by citrate reduction, the peak between 3269 and $3387 \mathrm{~cm}^{-1}$ was assigned to the O$\mathrm{H}$ stretching bond of citrate, that at $1591 \mathrm{~cm}^{-1}$ was attributed to the presence of $\mathrm{C}=\mathrm{O}$ stretching bonds, the peaks at 1403 and $830-881 \mathrm{~cm}^{-1}$ were assigned to $\mathrm{C}-\mathrm{H}$ bending groups, and the peak at $1121 \mathrm{~cm}^{-1}$ was due to the $\mathrm{C}-\mathrm{O}$ stretching bond [37]. On the other hand, loading the VAM onto AgNPs led to a peak shift of the aromatic $\mathrm{C}=\mathrm{C}$ stretching bonds from $1661 \mathrm{~cm}^{-1}$ to 1699 $\mathrm{cm}^{-1}$, and the $\mathrm{C}=\mathrm{O}$ bond stretch peaks also shifted from $1498 \mathrm{~cm}^{-1}$ to $1519 \mathrm{~cm}^{-1}$, which indicated that VAM had successfully conjugated with the AgNPs in the reaction system.

\subsection{Internalization of the AgNPs and AgNP-VAM conjugates}

To explore the internalization of AgNPs after conjugation with VAM, M. smegmatis cells were treated with AgNPs or AgNP-VAM conjugates and the real-time UV-vis absorption of these two biomaterials were monitored, as shown in Figure 3B. Compared with the cells treated with AgNPs, there was a significant reduction ( $15 \%)$ in the absorption intensity of the cells treated with AgNP-VAM for $1 \mathrm{~h}$ (Fig. 3B). Moreover, at the incubation time of $20 \mathrm{~h}$, the UVvis absorption value had dropped by 45\% in the AgNP@VAM-treated M. smegmatis cells, and the reduction was even more significant after $24 \mathrm{~h}$ of incubation. This indicated that the AgNPs had greatly improved the chances of entry of the conjugated VAM to the M. smegmatis cell, as proven by the remarkable decease in the UV-vis absorbance. Furthermore, this result 
demonstrates that AgNPs are very active drug carriers for loading the VAM effectively into $M$. smegmatis, therefore enabling enhanced internalization of the AgNP-VAM conjugate into the cells as compared with that of free AgNPs.

\subsection{Viability of M. smegmatis cells after treatment with AgNPs, AgNP-VAM conjugates, and VAM}

To investigate the potential effect of the AgNP-VAM conjugate on M. smegmatis, as well as of the sensitivity of VAM after its conjugation with AgNPs, the viability of $M$. smegmatis cells was evaluated. The kinetic growth curves of $M$. smegmatis in LB medium are shown in the presence of culture medium alone, AgNPs, AgNP-VAM conjugate, or free VAM solution (Fig.

4). Compared with that of the control and free AgNP-treated cells, the viability of bacteria treated with VAM alone or AgNP-VAM conjugate had decreased according to the incubation time (Fig. 4B). Moreover, free VAM at a concentration as low as $7.3 \mu \mathrm{g} / \mathrm{mL}$ led to the complete inhibition of $M$. smegmatis growth over an $8 \mathrm{~h}$ cultivation, whereas the inhibitory concentration of the AgNP-VAM conjugate was $54 \mu \mathrm{g} / \mathrm{mL}$, which contain approaximately 20ng of VAM, indicating a much lower viability of $M$. smegmatis compared with that seen with free VAM. It is to be noted that the amount of VAM loaded onto the AgNPs was the same as that in the freeVAM solution, allowing us to make a direct comparision between the AgNP-VAM conjugate and free VAM. The evaluation above indicated that the AgNP-VAM conjugate was more potent then the free VAM against $M$. smegmatis. A possible explanation for this activity enhancement by AgNP-VAM conjugation may be the improved internalization of the VAM-modified AgNPs by the endocytosis mechanism [33]. This potential of enhanced internalization of the AgNPVAM conjugate may help in the development of other intermittent treatment regimens with reduced drug doses for better patient compliance, thus improving the general outcome of 
therapy. In addition, the increased internalization of the VAM particles via bioconjugation translates to increased efficacy of the drug against $M$. smegmatis. All these assessments were constant with the UV-vis absorption results and TEM observation.

\subsection{TEM images of $M$. smegmatis after treatment with AgNPs, AgNP-VAM conjugates, and VAM}

The untreated $M$. smegmatis cell exhibited a typical rod-like shape, with a well-defined cell wall and evenly distributed interior content, indicating structural integrity (Fig. 5A). $M$. smegmatis cells treated with AgNPs also showed an intact cellular structure and membrane, with the AgNPs located randomly inside and outside of the cells (Fig. 5B), indicating that the free nanoparticles do not interact with the bacterial cells in any specific manner. On the other hand, significant morphological changes were observed in the $M$. smegmatis cells after exposure to free VAM or AgNP-VAM conjugate for $4 \mathrm{~h}$ (Fig. 5C and 5D), indicating specific interaction between theAgNP-VAM biomolecules and the bacteria. Some of the M. smegmatis cells were seriously damaged, the outer membrane was no longer intact, and interior substances, including the internalized AgNPs, had started to leak out (Fig. 5D). The damage was more severe with a longer treatment time with AgNP-VAM .

Our study revealed that the inactivation and associated damage of $M$. smegmatis should be largely attributed to the strong interaction between the AgNP-VAM conjugate and the $M$. smegmatis cell. The damage of the M. smegmatis cell wall in presence of AgNP-VAM the conjugate may be due to the strong binding affinity of the AgNPs to the cell membrane. This resulted in the successful internalization of the AgNP-VAM conjugate, thus leading to the successful drug loading of VAM into the M. smegmatis cell. The drug action then led to cell death and the subsequent discharge of the intracellular substances into the surrounding medium, 
following which the entire cell wall was destroyed and collapsed into the unstructured pieces. It should be noted that the underlying mechanism of internalization of the AgNP-VAM conjugate (i.e., regarding the interactions between the AgNP-VAM conjugate and $M$. smegmatis cell) is still not clear. One possible explanation is the ease with which AgNPs are internalized into M. smegmatis, a fact supported by the TEM images (Fig. 5B). The AgNPVAM conjugate also exhibited strong interactions with $M$. smegmatis by means of the strong affinity of the AgNPs to the cell wall. It is worth stressing that these observations and explanations are still preliminary, and further studies on the mechanism of the internalization as well as more microbial experiments on a molecular basis are definitely needed.

In conclusion, we have reported a simple method for the synthesis of AgNPs by using citrate as both the reducing and stabilizing agent. With the assistance of EDC and NHS, we have successfully loaded the macromolecular drug VAM onto the surface of the AgNPs and demonstrated enhanced internalization of the AgNP-VAM conjugate into the bacterial cells. The internalization capability of the conjugate was much better than that of bare AgNPs and free VAM. The improved internalization led to a significantly enhanced delivery of the macromolecular drug into the M. smegmatis cell, allowing for better efficacy of the drug to induce bacterial cell death, AgNP-VAM as evidenced by the UV, TEM, and confocal microscopic analyses. Based on the enhanced internalization of the AgNP-drug bioconjugate, the current research provides a new potential drug delivery system for the treatment of Mycobacterium-caused diseases such as tuberculosis 


\section{Declarations}

\section{Ethics approval and consent to participate}

No applicable

\section{Authors' contributions}

Fangfang Sun, Sangjin Oh and Jeonghyo Kim designed and performed the experiments, Tatsuya Kato and Hwa-Jung Kim helped analyze data, and Jaebeom Lee and Enoch Y. Park are the corresponding authors who designed the study, prepared the manuscript, provided funding support and contributed equally. All authors read and approved the final manuscript.

\section{Consent for publication}

This manuscript is approved by all authors for the submission.

\section{Availability of data and material}

The datasets during and/or analyzed during the current study are available from the corresponding author on reasonable request.

\section{Competing interests}

The authors declare that they have no competing.

\section{Funding}

This research was supported by a grant of the Korea Health Technology R\&D Project through the Korea Health Industry Development Institute (KHIDI), funded by the Ministry of Health \& Welfare, Republic of Korea (HI16C1553), the framework of international cooperation program managed by the National Research Foundation of Korea (NRF- 
2014K2A2A4001081), and the National Research Foundation of Korea (NRF) grant funded by the Korea government (MEST) (2016R1A2B4012072). This work was supported partly by Promotion of Nanobio-Technology Research to support Aging and Welfare Society from the Ministry of Education, Culture, Sports, Science and Technology, Japan. 


\section{Reference List}

1. Mathuria JP. 2009. Nanoparticles in tuberculosis diagnosis, treatment and prevention: a hope for the future. Digest Journal of Nanomaterials and Biostructures. 4: 309-312.

2. Kell AJ, Stewart G, Ryan S, Peytavi R, Boissinot M, Huletsky A, et al. 2008. Vancomycinmodified nanoparticles for efficient targeting and preconcentration of Gram-positive and Gram-negative bacteria. Acs Nano. 2: 1777-1788.

3. Cheon, S. A., Cho, H. H., Kim, J., Lee, J., Kim, H. J., \& Park, T. J. 2016. Recent tuberculosis diagnosis toward the end TB strategy. Journal of microbiological methods, 123, 51-61.

4. Shim BS, Chen W, Doty C, Xu C, Kotov NA. 2008. Smart electronic yarns and wearable fabrics for human biomonitoring made by carbon nanotube coating with polyelectrolytes. Nano letters. 8: 4151-4157.

5. Liong M, Lu J, Kovochich M, Xia T, Ruehm SG, Nel AE, et al. 2008. Multifunctional inorganic nanoparticles for imaging, targeting, and drug delivery. ACS nano. 2: 889-896.

6. Zhou, H., Zou, F., Koh, K., \& Lee, J. 2014. Multifunctional magnetoplasmonic nanomaterials and their biomedical applications. Journal of biomedical nanotechnology, 10(10), 29212949..

7. Perez JM, Simeone FJ, Saeki Y, Josephson L, Weissleder R. 2003. Viral-induced self-assembly of magnetic nanoparticles allows the detection of viral particles in biological media. Journal of the American Chemical Society. 125: 10192-10193.

8. Kim JS, Kuk E, Yu KN, Kim J-H, Park SJ, Lee HJ, et al. 2007. Antimicrobial effects of silver nanoparticles. Nanomedicine: Nanotechnology, Biology and Medicine. 3: 95-101.

9. Anandhakumar S, Raichur AM. 2013. Polyelectrolyte/silver nanocomposite multilayer films as multifunctional thin film platforms for remote activated protein and drug delivery. Acta biomaterialia. 9: 8864-8874.

10. Chen X, Schluesener H. 2008. Nanosilver: a nanoproduct in medical application. Toxicology letters. 176: 1-12.

11. Lok C-N, Ho C-M, Chen R, He Q-Y, Yu W-Y, Sun H, et al. 2006. Proteomic analysis of the mode of antibacterial action of silver nanoparticles. Journal of Proteome research. 5: 916924.

12. Wang H, Liu J, Wu X, Tong Z, Deng Z. 2013. Tailor-made Au@ Ag core? shell nanoparticle $2 \mathrm{D}$ arrays on protein-coated graphene oxide with assembly enhanced antibacterial activity. Nanotechnology. 24: 205102.

13. Duran N, Marcato PD, De Souza GI, Alves OL, Esposito E. 2007. Antibacterial effect of silver nanoparticles produced by fungal process on textile fabrics and their effluent treatment. Journal of biomedical nanotechnology. 3: 203-208.

14. Elechiguerra JL, Burt JL, Morones JR, Camacho-Bragado A, Gao X, Lara HH, Yacaman MJ. 2005. Interaction of silver nanoparticles with HIV-1. Journal of nanobiotechnology. 3: 1.

15. Lee J-S, Lytton-Jean AK, Hurst SJ, Mirkin CA. 2007. Silver nanoparticle-oligonucleotide 
conjugates based on DNA with triple cyclic disulfide moieties. Nano letters. 7: 2112-2115.

16. Thompson DG, Enright A, Faulds K, Smith WE, Graham D. 2008. Ultrasensitive DNA detection using oligonucleotide-silver nanoparticle conjugates. Analytical chemistry. 80: 2805-2810.

17. Ting BP, Zhang J, Gao Z, Ying JY. 2009. A DNA biosensor based on the detection of doxorubicin-conjugated Ag nanoparticle labels using solid-state voltammetry. Biosensors and Bioelectronics. 25: 282-287.

18. Ren $X$, Meng X, Chen D, Tang F, Jiao J. 2005. Using silver nanoparticle to enhance current response of biosensor. Biosensors and Bioelectronics. 21: 433-437.

19. Pal S, Tak YK, Song JM. 2007. Does the antibacterial activity of silver nanoparticles depend on the shape of the nanoparticle? A study of the gram-negative bacterium Escherichia coli. Applied and environmental microbiology. 73: 1712-1720.

20. Aymonier C, Schlotterbeck U, Antonietti L, Zacharias P, Thomann R, Tiller JC, Mecking S. 2002. Hybrids of silver nanoparticles with amphiphilic hyperbranched macromolecules exhibiting antimicrobial properties. Chemical Communications. 3018-3019.

21. Rubio-Martínez M, Puigmartí-Luis J, Imaz I, Dittrich PS, Maspoch D. 2013. "Dual-Template" Synthesis of One-Dimensional Conductive Nanoparticle Superstructures from Coordination Metal-Peptide Polymer Crystals. Small. 9: 4160-4167.

22. Maretti L, Billone PS, Liu Y, Scaiano JC. 2009. Facile photochemical synthesis and characterization of highly fluorescent silver nanoparticles. Journal of the American Chemical Society. 131: 13972-13980.

23. Huang H, Ni X, Loy G, Chew C, Tan K, Loh F, et al. 1996. Photochemical formation of silver nanoparticles in poly ( $\mathrm{N}$-vinylpyrrolidone). Langmuir. 12: 909-912.

24. Yin B, Ma H, Wang S, Chen S. 2003. Electrochemical synthesis of silver nanoparticles under protection of poly (N-vinylpyrrolidone). The Journal of Physical Chemistry B. 107: 88988904.

25. Rodriguez-Sanchez L, Blanco M, Lopez-Quintela M. 2000. Electrochemical synthesis of silver nanoparticles. The Journal of Physical Chemistry B. 104: 9683-9688.

26. Biswal J, Misra N, Borde LC, Sabharwal S. 2013. Synthesis of silver nanoparticles in methacrylic acid solution by gamma radiolysis and their application for estimation of dopamine at low concentrations. Radiation Physics and Chemistry. 83: 67-73.

27. Hu B, Wang S-B, Wang K, Zhang M, Yu S-H. 2008. Microwave-assisted rapid facile "green" synthesis of uniform silver nanoparticles: self-assembly into multilayered films and their optical properties. The Journal of Physical Chemistry C. 112: 11169-11174.

28. Pillai ZS, Kamat PV. 2004. What factors control the size and shape of silver nanoparticles in the citrate ion reduction method? The Journal of Physical Chemistry B. 108: 945-951.

29. Turkevich J, Stevenson PC, Hillier J. 1951. A study of the nucleation and growth processes in the synthesis of colloidal gold. Discussions of the Faraday Society. 11: 55-75.

30. Zhang, T., Wang, L., Chen, Q., \& Chen, C. 2014. Cytotoxic potential of silver nanoparticles. 
Yonsei medical journal, 55(2); 283-291.

31. Burello, E., \& Worth, A. P. 2011. QSAR modeling of nanomaterials. Wiley Interdisciplinary Reviews: Nanomedicine and Nanobiotechnology, 3(3); 298-306.

32. Puzyn, T., Rasulev, B., Gajewicz, A., Hu, X., Dasari, T. P., Michalkova, A., ... \& Leszczynski, J. 2011. Using nano-QSAR to predict the cytotoxicity of metal oxide nanoparticles. Nature nanotechnology, 6(3); 175-178.

33. Hubbard BK, Walsh CT. 2003. Vancomycin assembly: nature's way. Angewandte Chemie International Edition. 42: 730-765.

34. Walsh C. 1999. Deconstructing vancomycin. Science. 284: 442-443.

35. Everaerts F, Torrianni M, Hendriks M, Feijen J. 2008. Biomechanical properties of carbodiimide crosslinked collagen: influence of the formation of ester crosslinks. Journal of Biomedical Materials Research Part A. 85: 547-555.

36. Loveymi BD, Jelvehgari M, Zakeri-Milani P, Valizadeh H. 2012. Design of vancomycin RS-100 nanoparticles in order to increase the intestinal permeability. Advanced pharmaceutical bulletin. 2: 43.

37. Thottoli AK, Unni AKA. 2013. Effect of trisodium citrate concentration on the particle growth of ZnS nanoparticles. Journal of Nanostructure in Chemistry. 3: 1-12.

38. Yoo HS, Lee $\mathrm{KH}$, Oh JE, Park TG. 2000. In vitro and in vivo anti-tumor activities of nanoparticles based on doxorubicin-PLGA conjugates. Journal of Controlled Release. 68: 419-431. 


\section{Legends of figures}

Figure 1. Schematic representation of the drug@AgNP bioconjugation pathway (A), internalization of the macromolecular drug in Mycobacterium smegmatis with the assistance of AgNPs (B), and the molecule structure of vancomycin (C). EDC, 1-ethyl-3-(3dimethylaminopropyl) carbodiimide; NHS, n-hydroxysuccinimide; 8-MOA, 8mercaptootanoic acid.

Figure 2. UV-visible absorption spectra of the prepared (A) AgNPs and (B) AgNP-VAM conjugate. The inset in A is a digital image of the synthesized AgNPs. (C) and (D) TEM images of the synthesized AgNPs and AgNP-VAM conjugates.

Figure 3. (A) FTIR spectra of the synthesized AgNPs, AgNP-VAM conjugate, and vancomycin (VAM). (B) UV-visible absorption spectra of Mycobacterium smegmatis at 420 nm according to incubation time, after treatment with AgNPs or AgNP-VAM conjugate.

Figure 4. (A) Viability test of Mycobacterium smegmatis after treatment with AgNPs, AgNPVAM conjugate, or vancomycin (VAM). (B) Quantification of M. smegmatis (CFU/mL) according to the incubation time, after treatment with AgNPs, AgNP-VAM conjugate, or VAM.

Figure 5. Confocal microscopy image of Mycobacterium smegmatis cells (A), and TEM images of M. smegmatis after treatment with (B) AgNPs, (C) vancomycin (VAM), and (D) AgNP-VAM conjugate for $4 \mathrm{~h}$. 

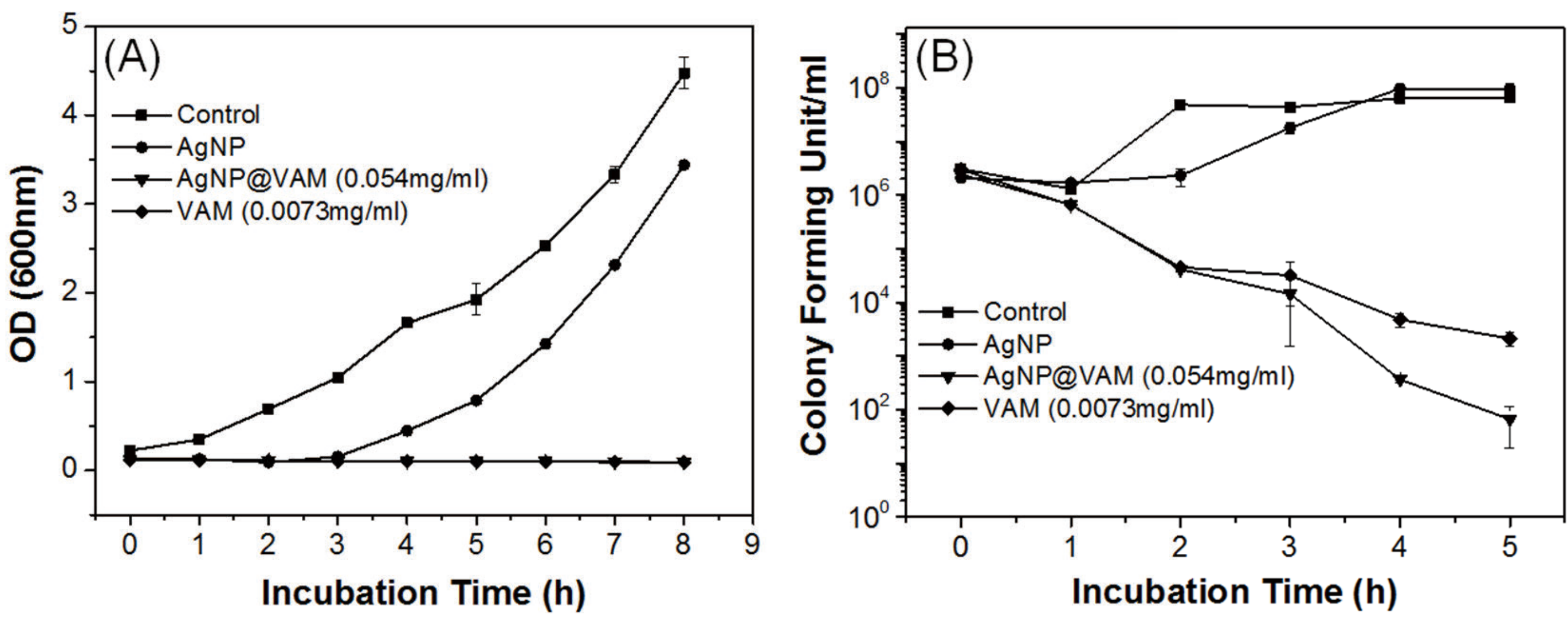
